\title{
RESULTADO DA REPARAÇÃO DO MANGUITO ROTADOR EM LESÕES DO TIPO C1 E C2 DE SNYDER, CONSIDERANDO FUMANTES E NÃO FUMANTES
}



Sérgio Correa Pinto Júnior ${ }^{1}$, Lessandro Gesser Luciano', Crischiman Dal Zotto², Felipe Fantozzi Vieira', Ralf Klassen², Airton Rodrigues ${ }^{3}$, Francisco José dos Santos Neto 3

\section{RESUMO}

Objetivo: Avaliar a influência do tabagismo nos resultados cirúrgicos do reparo das lesões completas do manguito rotador tipos C1 e C2 de Snyder. Métodos: Foram avaliados 166 pacientes que haviam sido submetidos a tratamento cirúrgico para lesão completa do manguito rotador tipo C1 e C2 de Snyder, entre junho de 2002 a dezembro de 2006. Foram considerados critérios de inclusão um seguimento mínimo de 24 meses e ausência de cirurgias prévias no ombro acometido. Excluíram-se os pacientes que apresentavam outras lesões associadas. Foram avaliados os paciente fumantes e não fumantes de acordo com os critérios da Organização Mundial da Saúde (OMS). Houve predomínio de pacientes do sexo feminino (119) em relação ao masculino (47), e a idade média foi de 57 anos (38 a 78). Do total de 166 pacientes avaliados, foram considerados fumantes 21 pacientes e não fumantes 145. Os resultados finais foram avaliados pelos critérios da UCLA (University of California at Los Angeles) e a análise estatística foi feita pelo programa Epi Info ${ }^{\circledR}$. Resultados: Pelos critérios da UCLA, os pacientes fumantes tiveram uma média final de 32,6 pontos, enquanto os não fumantes de 33,8. Na análise estatística pós-operatória houve diferença entre os dois grupos, com os pacientes não fumantes tendo melhor resultado final. Conclusão: O tabagismo interfere no resultado final das reparações de lesões pequenas e médias do manguito rotador.

Descritores - Artroscopia; Ombro/lesões; Ombro/cirurgia; Dor de Ombro

\section{ABSTRACT}

Objective: To evaluate the influence of smoking on the results of surgical repair of complete lesions of the rotator cuff Snyder types C1 and C2. Methods: We studied 166 patients who had undergone surgical treatment for complete lesion of the rotator cuff Snyder type C1 and C2, from June 2002 to December 2006. Inclusion criteria were a minimum follow-up period of 24 months and the absence of previous surgery on the affected shoulder. Patients with other associated injuries were excluded. We evaluated the smoking and nonsmoking patients according to the criteria of the World Health Organization (WHO). More patients were female (119) than male (47), and the mean age was 57 years (38 to 78). Of the 166 patients evaluated, 21 were classified as smokers and 145 as nonsmokers. The final results were evaluated by the UCLA (University of California at Los Angeles) and statistical analysis was performed using Epi Info ${ }^{\circledR}$. Results: According to the UCLA criteria, smokers had a final average of 32.6 points, while non-smokers had 33.8. Postoperative statistical analysis revealed a difference between the two groups, with non-smoking patients having a better outcome. Conclusion: Smoking interferes with the final results of the repairs of small and medium-sized lesions of the rotator cuff.

Keywords - Arthroscopy; Shoulder/injuries; Shoulder/surgery; Shoulder Pain

\footnotetext{
1 - Ortopedista da Clínica do Ombro - Florianópolis (SC), Brasil.

2 - Ortopedista do Instituto de Ortopedia e Traumatologia (IOT) - Blumenau (SC), Brasil.

3 - Ortopedista do Centro de Estudos Ortopédicos de Passo Fundo (CEOP) - Passo Fundo (RS), Brasil.
}

Trabalho realizado na Clínica do Ombro (Florianópolis - SC).

Correspondência: Rua Presidente Coutinho, 579 - Sala 603, Centro - 88015-230 - Clínica do Ombro - Florianópolis (SC), Brasil. E-mail: sergiopintojr@gmail.com

Trabalho recebido para publicação: 22/09/09, aceito para publicação: 09/03/10. 


\section{INTRODUÇÃO}

O tabagismo é considerado um problema de saúde mundial, afetando vários sistemas orgânicos, e suas consequências foram avaliadas por várias especialidades médicas. Poucos estudos tiveram o objetivo de relacionar os efeitos do tabagismo com as lesões do manguito rotador. Sabe-se que a borda da lesão do manguito rotador é hipovascular ${ }^{(1)}$, e pode-se suspeitar que usuários de cigarro teriam uma diminuição ainda maior dessa vascularização. Mallon et al $^{(2)}$ encontraram resultados pós-operatórios inferiores em pacientes tabagistas. Questiona-se se esses piores resultados seriam consequência de lesões pré-operatórias mais graves em pacientes tabagistas, já que Baumgarten et $a l^{(3)}$ demonstraram que fumantes tendem a ter lesões maiores que não fumantes. Nosso estudo propõe-se a responder essa questão avaliando pacientes com lesões de tamanho semelhante no pré-operatório.

\section{Objetivo}

Avaliar a influência do tabagismo nos resultados cirúrgicos do reparo das lesões completas do manguito rotador, tipos C1 e C2 de Snyder et $a l^{(4)}$.

\section{MATERIAL E MÉTODO}

De junho de 2002 a dezembro de 2006 foram realizadas 1.291 videoartroscopias para manguito rotador pelo mesmo serviço. Foram incluídos 166 pacientes com lesões classificadas como tipo C1 e C2 da classificação de Snyder et $a l^{(4)}$. Nesse critério, as lesões articulares estão no grupo A, as bursais no B, e as completas no C. Snyder et al descreveram C1 como uma lesão pequena, completa puntiforme, e C2 como moderada (geralmente menor que $2 \mathrm{~cm}$ ), acometendo apenas um tendão, sem retração. Foram considerados critérios de inclusão um seguimento mínimo de 24 meses e ausência de cirurgia prévia no ombro operado. Excluíram-se os pacientes que apresentavam outras lesões associadas, como os acromiale, luxação glenoumeral, SLAP lesion, tendinite calcária e lesão do tendão da cabeça longa do bíceps. Foram considerados apenas os pacientes fumantes e não fumantes, de acordo com os critérios da Organização Mundial da Saúde (OMS) ${ }^{(5)}$, sendo que pacientes extabagistas e fumantes leves foram excluídos. Do total de 166 pacientes avaliados, foram considerados fumantes 21 (13\%) pacientes e não fumantes 145 (87\%). O seguimento médio foi de 34 meses (24 a 78). Houve predomínio do sexo feminino (119 pacientes; 72\%) em relação ao masculino (47 pacientes; 28\%), e a idade média foi de 57 anos (38 a 78 anos).

As videoartroscopias foram realizadas em decúbito lateral sob tração no membro operado. Foram utilizadas âncoras de titânio, dispostas em fileira única, e com pontos simples. Todas as lesões do manguito rotador foram completamente fechadas, e a acromioplastia executada em todos os casos. Os dois grupos de pacientes foram submetidos à mesma rotina pós-operatória, com seis semanas de tipoia, seguida de ganho de mobilidade e reforço muscular.

Os resultados finais foram avaliados pelos critérios da UCLA (University of California at Los Angeles) ${ }^{(6)}$, que pontua critérios de dor (1-10), função (1-10), elevação (0-5), força (0-5) e satisfação (0 ou 5) para agrupar estes resultados em excelentes (34 e 35 pontos), bons (28 a 33), razoáveis (21 a 27) e ruins (20 ou menos). Foi utilizado o programa Epi Info ${ }^{\circledR}$ (teste de KruskallWallis; $\mathrm{p}<0,04)$ para a análise estatística.

\section{RESULTADOS}

O número médio de âncoras variou de um a três nos dois grupos, com média de 1,3 âncoras no grupo dos tabagistas e 1,1 para os não fumantes. Não houve diferença estatística quanto ao número de âncoras. Os pacientes tabagistas evoluíram de um UCLA pré-operatório de 15,6 (mínimo: 10; máximo: 21) para um pós-operatório de 32,6 (mínimo: 26; máximo: 35). Os não fumantes evoluíram de um UCLA de 16 (mínimo: 11; máximo: 21) para 33,8 (mínimo: 28; máximo: 35) (Figura 1).

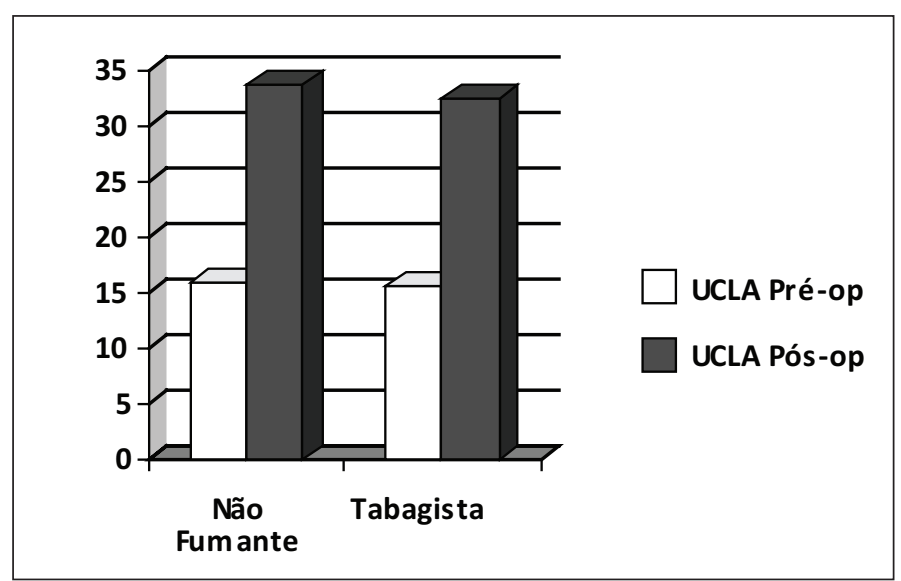

Figura 1 - UCLA pré e pós-operatório.

Levando-se em conta os valores considerados excelentes pelos critérios da UCLA (34 e 35 pontos), 63\% dos pacientes não fumantes enquadraram-se neste grupo, 
enquanto que $47,6 \%$ dos tabagistas encontraram-se no mesmo grupo, na avaliação pós-cirúrgica. Na análise estatística (Epi Info ${ }^{\circledR}$; teste de Kruskall-Wallis com p $<0,04)$ não houve diferença significativa entre os dois grupos no pré-operatório. Considerando-se os dados pós-operatórios, houve diferença entre os dois grupos ( $p=0,0094$ ), com os pacientes não fumantes tendo melhor resultado final.

\section{DISCUSSÃO}

Apesar de amplamente estudado por outras áreas médicas, o efeito do cigarro na ortopedia ${ }^{(7)}$ ainda é escasso. Especificamente na área do ombro, poucos estudos foram publicados. Galatz et $a l^{(8)}$ utilizaram ratos randomicamente submetidos à bomba de infusão salina ou nicotina, e o grupo com nicotina teve piores resultados mecânicos do reparo do manguito rotador. Kane et $a l^{(9)}$ encontraram um índice maior de rotura de manguito rotador em cadáveres de pacientes fumantes. Mallon et $a l^{(2)}$, em uma análise de cirurgias abertas, encontrou piores resultados em fumantes. Em um estudo com ultrassonografias, Baumgarten et $a l^{(3)}$, além de diagnosticarem mais lesões do manguito rotador em fumantes, encontraram lesões maiores nesse grupo. Mesmo com esses estudos, uma questão persistiu: os resultados inferiores seriam decorrência direta do cigarro, ou secundários às lesões maiores que os tabagistas tendem a apresentar? Para responder a esta pergunta sem que essa diferença pudesse causar um viés na avaliação dos resultados, equalizamos os dois grupos, analisando apenas pacientes com lesões pequenas e médias, de acordo com a classificação de Snyder et $a l^{(4)}$. Levamos também em consideração os critérios da OMS para tabagismo, pois parece haver uma confusão nos critérios do uso de cigarro, com cada autor utilizando seu próprio método para classificar o

\section{REFERÊNCIAS}

1. Godinho GG, Freitas JMA, França FO, Filho JA, Schio C, Pinto SC. Estudo da vascularização das bordas das lesões nas roturas completas do manguito rotador. Rev Bras de Ortop. 2007;42(6)161-8.

2. Mallon WJ, Misamore G, Snead DS, Denton P. The impact of preoperative smoking habits on the results of rotator cuff repair. J Shoulder Elbow Surg. 2004;13(2):129-32.

3. Baumgarten KM, Gerlach D, Galatz LM, Teefey SA, Middleton WD, Ditsios K et al. Cigarette smokers increases the risk for rotator cuff tears. Clin Orthop Relat Res. 2010;468(6):1534-41.

4. Snyder SJ, Karzel RP, Del Pizzo W, Ferkel RD, Friedman MJ. SLAP lesions of the shoulder. Arthroscopy. 1990;6(4):274-9.

5. Organização Mundial da Saúde (OMS). Guidelines for the conduct of tobacco tabagismo. Com todos os critérios de exclusão utilizados, reduzimos muito o grupo inicialmente operado (1.291) para o avaliado (166), mas consideramos que criamos dois grupos homogêneos, em que o único diferencial foi a utilização ou não de cigarro. Não houve diferença estatística quanto ao número de âncoras. Não houve diferença estatística na comparação dois grupos pré-operatórios, mas esta diferença foi significativa no pós-operatório. Esses resultados ratificam as conclusões de Mallon et $a l^{(2)}$, que tabagistas têm resultados inferiores quando comparados a não fumantes, mesmo quando avaliamos apenas lesões de mesmo tamanho. Na comparação dos dois estudos, porém, a diferença estatística pós-operatória foi maior no trabalho de Mallon et $a l^{(2)}$, com não fumantes tendo resultados finais semelhantes ao nosso estudo, mas tabagistas tendo resultados muito inferiores. Consideramos que este fato seja pela tendência que os fumantes têm de sofrer lesões maiores, e essas lesões foram avaliadas por aquele estudo, mas não pelo nosso. Na análise dos motivos que levam a piores resultados nos tabagistas, considera-se que a nicotina causa redução do calibre vascular, diminuição da produção de fibroblastos e uma hipóxia tecidual, com diminuição da proliferação celular ${ }^{(8)}$. Com isso, sugerimos três cuidados ao operar um paciente tabagista: (1) solicitar ao paciente que diminua o uso de cigarro (de preferência a interrupção total); (2) no transoperatório, debridar a borda da lesão do manguito rotador ${ }^{(1)}$, levando uma zona de melhor vascularização para de encontro com o osso; e (3) considerar a possibilidade de um maior período de imobilização pós-operatória nesses pacientes.

\section{CONCLUSÃO}

O tabagismo interfere no resultado final das reparações de lesões pequenas e médias do manguito rotador.

smoking among health professionals. Report WHO. Meeting Winnipeg. Canada. 7-9 de julho, 1983.

6. Ellman H, Hanker G, Bayer M. Repair of the rotator cuff: end-result study of factors influencing reconstruction. J Bone Joint Surg Am. 1986;68(8):1136-44.

7. Mosley LH, Finseth F. Cigarette smoking: impairment of digital blood flow and wound healing in the hand. Hand. 1977;9(2):97-101.

8. Galatz LM, Silva MJ, Rothermich SY, Zaegel MA, Havlioglu N, Thomopoulos S. Nicotine Delays Tendon-to-Bone Healing in a Rat Shoulder Model. J Bone Joint Surg Am. 2006;88(9):2027-34.

9. Kane SM, Dave A, Haque A, Langston K. The incidence of rotator cuff disease in smoking and non-smoking patients: a cadaveric study. Orthopedics. 2006;29(4): 363-6. 